\title{
Screening for main components associated with the idiosyncratic hepatotoxicity of a tonic herb, Polygonum multiflorum
}

Chunyu $\mathrm{Li}^{1,2, *}$, Ming $\mathrm{Ni}^{1, *}$, Zhaofang $\mathrm{Bai}^{1{ }^{1, *}}$, Congen Zhang ${ }^{1}$, Yanling Zhao ${ }^{1}$, Ruiyu $\mathrm{Li}^{1}$, Can $\mathrm{Tu}^{1}$, Huifang $\mathrm{Li}^{7}$, Jing Jing ${ }^{3}$, Yakun Meng ${ }^{1}$, Zhijie Ma ${ }^{1,4}$, Wuwen Feng ${ }^{1}$, Jinfa Tang ${ }^{1}$, Yun Zhu ${ }^{3}$, Jinjie $\mathrm{Li}^{6}$, Xiaoya Shang ${ }^{6}$, Zhengsheng Zou ${ }^{5}$, Xiaohe Xiao $(\bowtie)^{3}$, Jiabo Wang $(\bowtie)^{1}$

${ }^{1}$ China Military Institute of Chinese Medicine, 302 Military Hospital, Beijing 100039, China; ${ }^{2}$ Institute of Medicinal Plant Development, Chinese Academy of Medical Sciences \&Peking Union Medical College, Beijing 100193, China; ${ }^{3}$ Integrative Medical Center, 302 Military Hospital, Beijing 100039, China; ${ }^{4}$ Beijing Friendship Hospital, Capital Medical University, Beijing 100050, China; ${ }^{5}$ Diagnosis and Treatment Center for Non-infectious Diseases, 302 Military Hospital, Beijing 100039, China; ${ }^{6}$ Beijing Union University, Beijing 100101, China; ${ }^{7}$ Shanxi University of Traditional Chinese Medicine, Taiyuan 030619, China

(C) Higher Education Press 2020

Erratum to Frontiers of Medicine 2017 11(2): 253-265

https://doi.org/10.1007/s11684-017-0508-9

The original version of this article unfortunately contained some mistakes.

The panel of "LPS"/drug" in Fig. 3B (Page 259), the panels of "LPS $/$ EtoAc $^{+}$" and "LPS" $/$Residue $^{+}$" in Supplementary Fig. 3 (Page 7 in the online supplementary materials) should be corrected. The authors apologize for this error and state that this does not change the scientific conclusions of the article in any way. The corrected figures are as following:

The online version of the original article can be found at https://doi.org/ 10.1007/s11684-017-0508-9

Correspondence: Xiaohe Xiao, pharmacy302xxh@126.com; Jiabo Wang, pharm_sci@126.com

${ }^{*}$ These authors contributed equally to this work. 
A

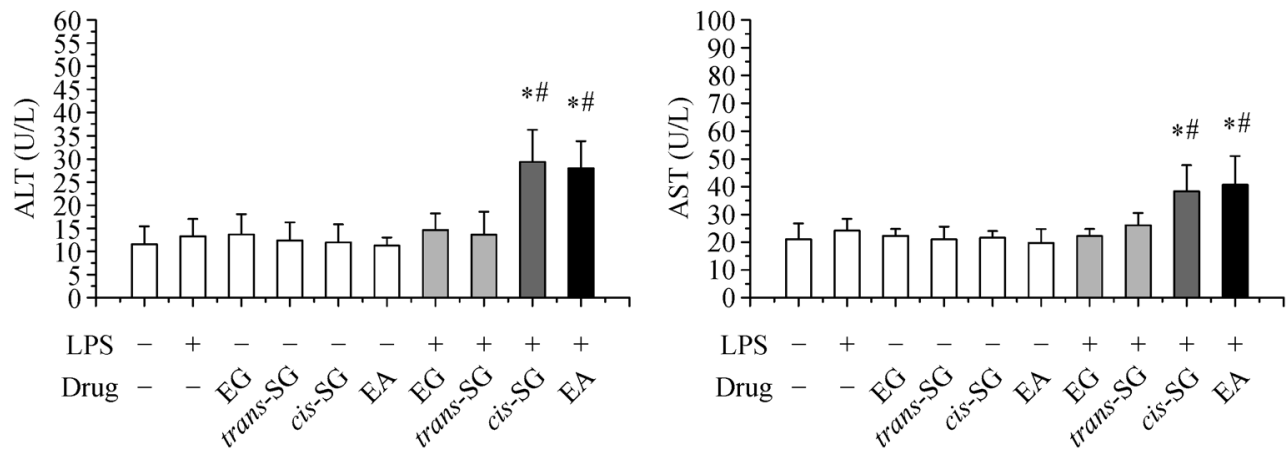

B
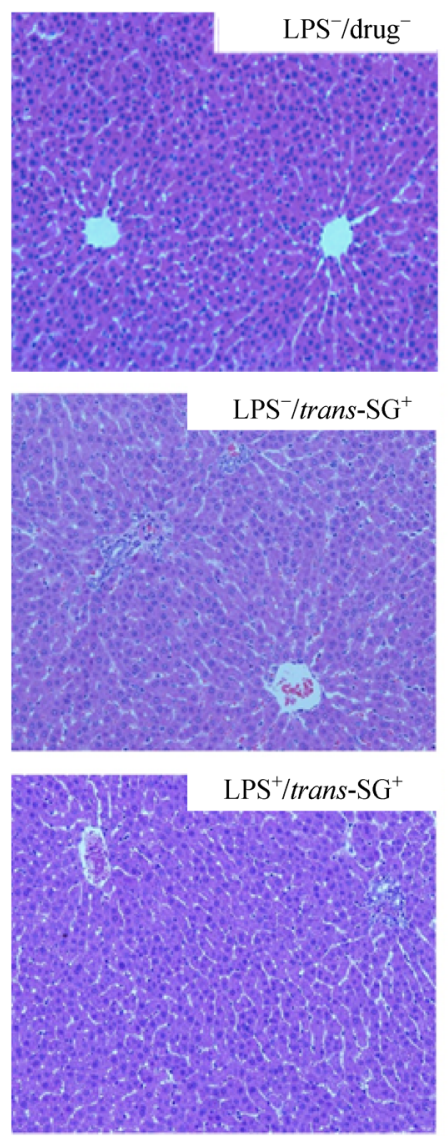
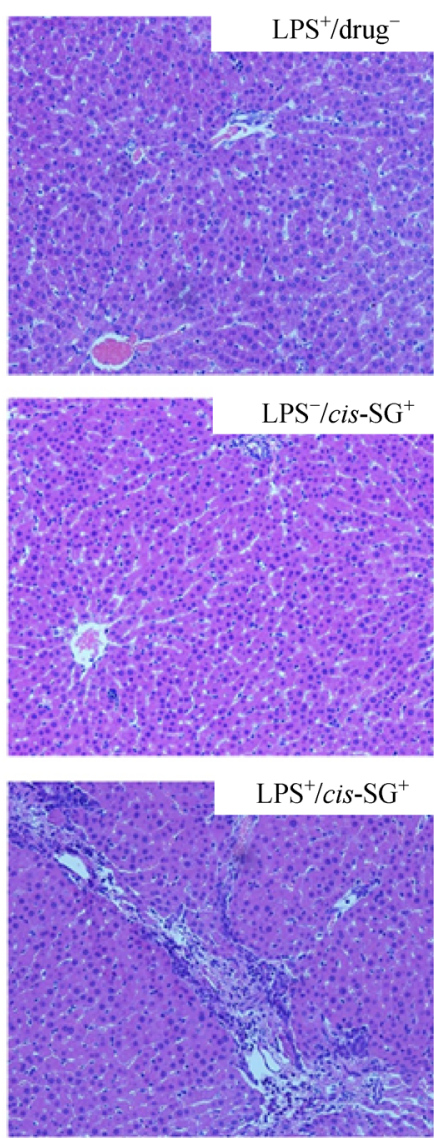

C
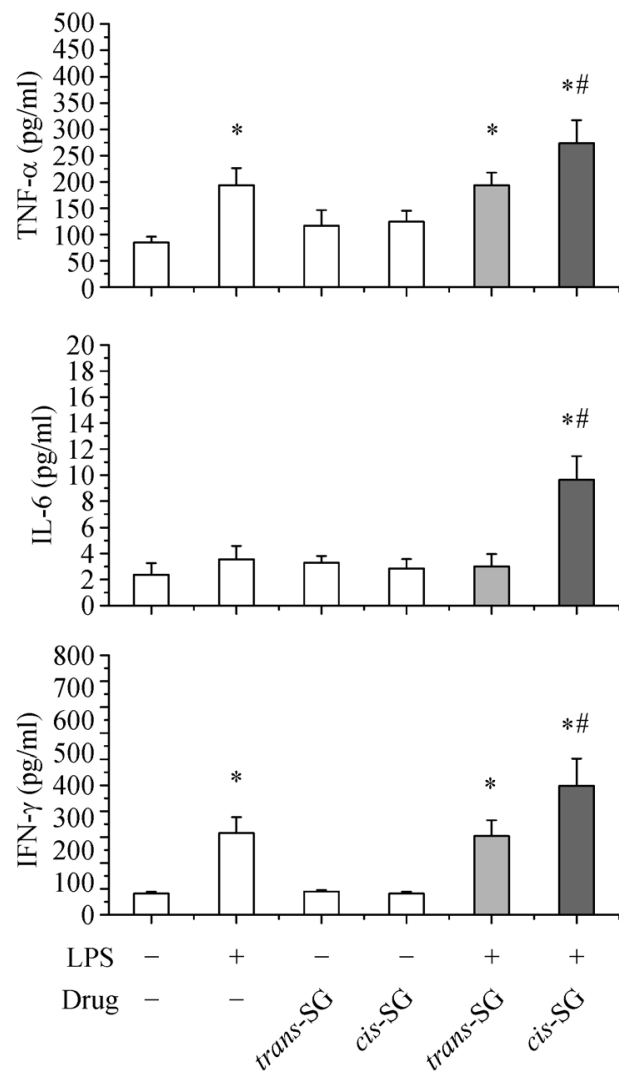

The corrected Fig. 3 

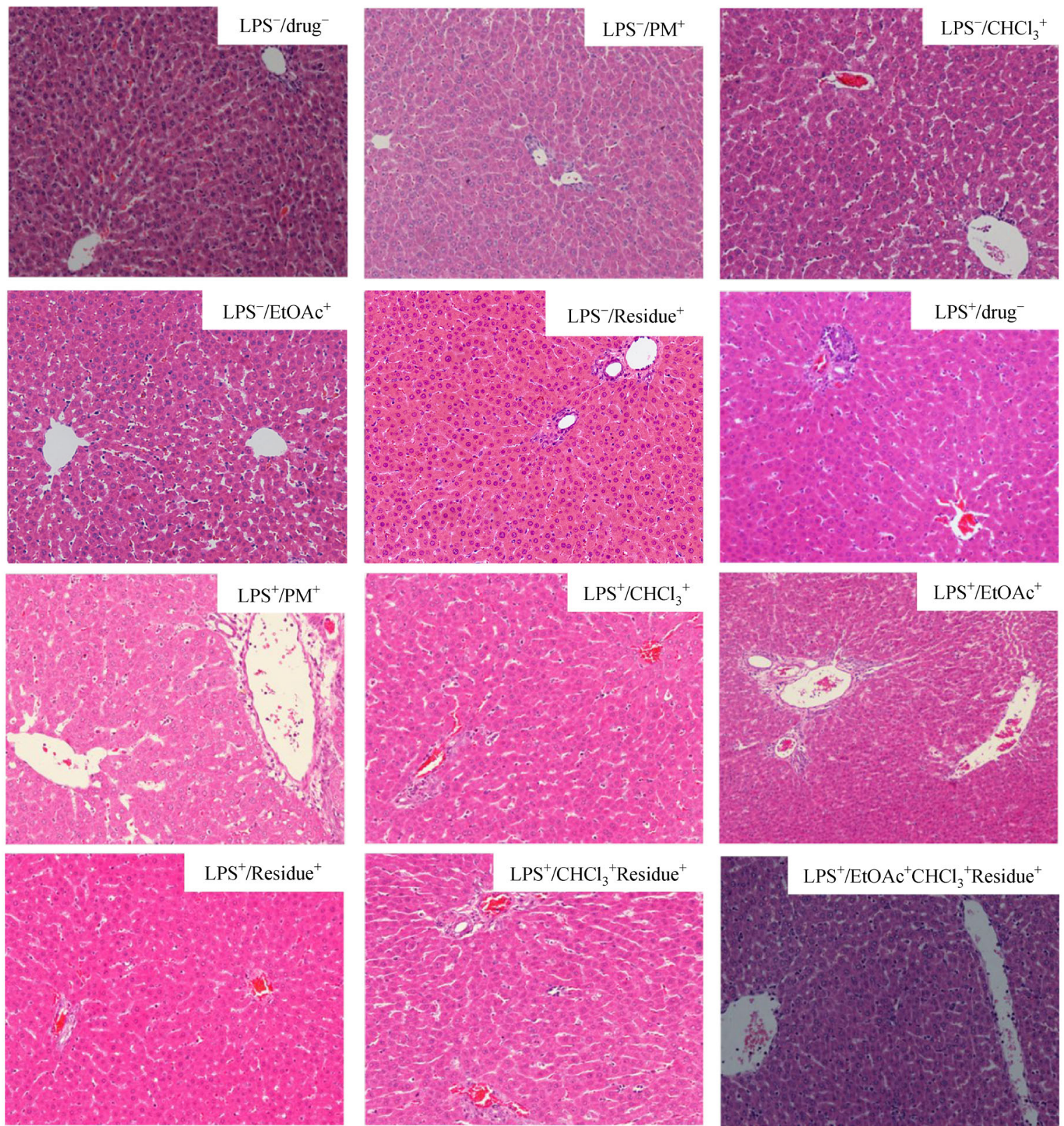

The corrected supplementary Fig. 3 